\title{
Survey of Various Methods used for Integrating Machine Learning into Brain Tumor Detection and Classification
}

\author{
Shaik Masood Ahamed \\ Research Scholar, Department of CSE, \\ Sathyabama Institute of Science and Technology, \\ Chennai, Tamilnadu 600119. India \\ masooda100@gmail.com \\ Dr. J. Jabez \\ Associate Professor, Department of CSE, \\ Sathyabama Institute of Science and Technology, \\ Chennai,Tamilnadu 600119, India \\ jabezme@gmail.com
}

\begin{abstract}
With the advancement of technology and ever-growing trough of data around the world there has been a fast increase in the accessibility of clinical databases and clinical symbolisms to everyone around the globe. The inaccuracy of the world in foreseeing oncoming and identifying present diseases before they worsen, from these data troughs has invited the network of researchers to soak up the challenges in this area. The crucial apprehensive system of homo sapiens is specially composed of the combination of the cerebrum, pons, cerebellum, encephalon which we in short call as the 'Brain'. This brain is then connected to the rest of the body via the Spinal cord. The human mind tosses and throws a wide variety of demanding situations and a great number of difficulties to the community of researchers. Machine Learning(ML) capabilities furnish machines with the capacity to become competent in domains without being clear-cut coded for the same. Data from various clinical sources was dissected adequately utilizing ML calculations and interpretations were made on the outcomes. Vital elements of the chosen research - wings of the healthcare system, mining of data, various kinds of analytics, information, data and statistical assets - were extricated to give a methodical perspective on advancements in this field and conceivable future bearings. Absence of prescriptive investigation in exercise and integration of domain professionals in the dynamic procedure, stresses the need of examination and research in the upcoming future.
\end{abstract}

In this paper we survey the various methods used for the implementation of ML backed technology into serving the needs of the medical field in both. Detection classification of a brain tumour from an MRI.

Keywords: Image processing, Segmentation, MRI (Magnetic Resonance Image), tumor.

\section{Introduction}

Brain tumour might be a group of anomalous cells developing inside the cerebrum. It may arise in any individual basically of any age group. It's going to even alter from one consultation to future ones, though its consequences may not be consistent for various patients undergoing treatment. Brain tumours appear at any region within the brain, in numerous different intensities, may range among being malignant and benign and may have a spread of sizes and compositions. The Benign type of tumours do not contain any cancerous cells and thus can be kept in check using modern radiology techniques or in most cases are carefully surgically pulverised. Malignant tumours on the other hand have a heterogeneous structure and comprise of dangerous cancer cells. Throughout thelength of this paper, we will in general try to actualize a strategy which may characterize various tumours and try to get extra accurate end results.

We will go stage wise in the process followed for this characterization according to Nilesh Bhaskarrao Bahadure, Arun Kumar Ray, Har Pal Thethi. [11] various methods and the comparison between these methods at the various stages of this process and state the optimum and efficient method to be chosen for the same. 


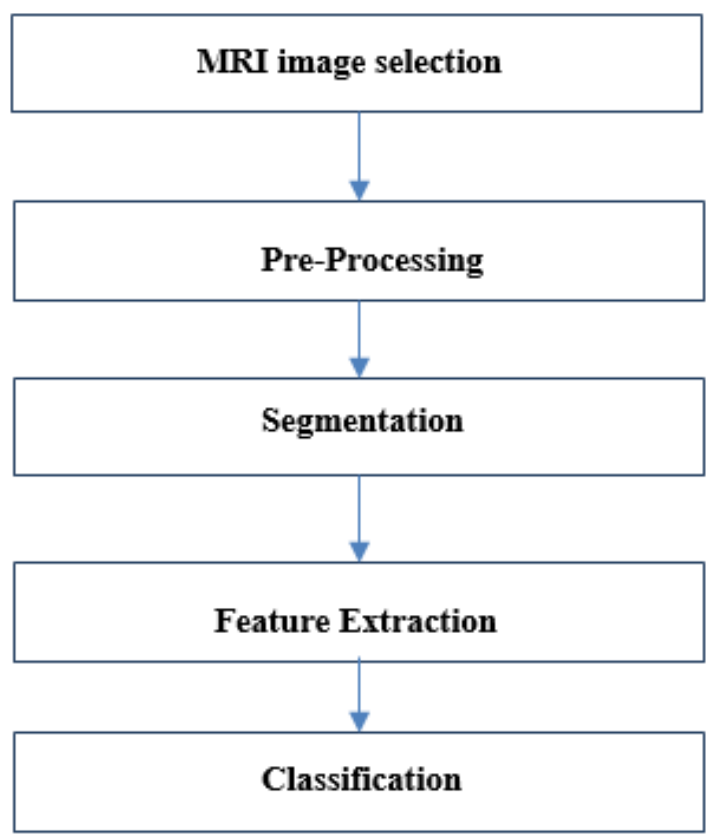

2. Methodology

\subsection{Image Selection}

For using the advanced capabilities offered by Machine learning technology, the most trivial component is the data. For our use case we provide MR images of the brain as the data set, as that is the organ in question of our research. An MRI is used compared to the other techniques namely X-rays and CT-scans because the former gives us more intimate information about the composition, blood supply and the entire anatomy of the organ. Thus this makes it easier to study and identify various anomalies in the organ and thus is the best choice of data set.

\subsection{Pre-processing}

Ample amount of top notch information is the way to an incredible machine learning model. However, great data sources are in shortage, and that shortage can hinder the advancement of a model. An approach to get around an absence of good information sources and good quality of data is to augment dataset.Brilliantactics when applied to deal with programmatic data augmentation might boom the dimensions of your training set. Furthermore, the model will frequently be increasingly powerful (and forestall overfitting) also, be less complex because of a superior training set.

The real-time MRIs which have not been handled may additionally include some commotion, and as a result, the image needs to be improved before making use of the algorithm to show higher overall efficiency. The intention of the pre-processing is to enhance the image quality that facilitates for further processing. Pre-processing enables decreasing the unpredictability and exponentially enhancing the scope of precision by enhancing the positive parameters of MR images including enhancing the signal-to-noise ratio, improving the visibility of MRIs, casting off the inappropriate noise and undesired elements from the background, smoothing the internal region, and retaining its edges.

There are numerous strategies available with a purpose to clear the noise and to acquire the clean image. The image enhancement relies upon various factors like computational time and price, data source quality, and the strategiesused for noise removal. Quite a few linear and non-linear techniques are used for the image preprocessing.

\subsection{Segmentation}

Segmentation can be defined as the division of the image into various regions based on attributes like intensity, gray level and constrast. As per Nilesh Bhaskarrao Bahadure, Arun Kumar Ray, Har Pal Thethi.[12] thus aim at getting connected components from the MRI in this step. Segmentation can be classified into four types and various methods.

2.3.1 Threshold-based segmentation: This is the grouping and division of regions of the MRI based on the brightness levels of the pixels after being pre-processed. Global, adaptive and local thresholding are the three further subcategories in this. 
2.3.2. Edge-based segmentation: Boundaries of the regions are identified by obtaining a binary picture studying the pixels close to the boundaries and observing their sudden transformation in brightness is the strategy used in this technique.

Eg: Gray Histogram Technique, Watershed segmentation and Gradient based method.

2.3.3 Region-based segmentation: Region growing, Region splitting and Region merging are the methods used in this technique to identify and segregate regions based on their similarity. This is not widely used because low accuracy associated with this method[14].

As it can be observed all the mentioned techniques follow the simple binary concept of similarity and discontinuity. The principle aim is however same for all the techniques- to separate and segregate the MRI based into various regions so it can be useful for the further steps[9].

\subsection{Feature Extraction}

This is the step in the process where the information from the image is extracted and sent for to the main algorithms for classification and data processing.

Many different properties are extracted here like:

2.4.1 Corelation: It is used to calculate the spatial dependencies between the pixels. Here $\sigma_{\mathrm{x}} \sigma_{\mathrm{y}}$ give the SD along the horizontal and vertical domains respectively and $\mathrm{M}_{\mathrm{x}}$ and $\mathrm{M}_{\mathrm{y}}$ give the mean along the horizontal and vertical domains

$$
C_{\text {orr }}=\frac{\sum_{x=0}^{m-1} \sum_{y=0}^{n-1}(x, y) \mathrm{f}(\mathrm{x}, \mathrm{y})-M \mathrm{x} M \mathrm{y}}{\sigma_{x} \sigma_{y}}
$$

2.4.2 Skewness: This is used to calculate the lack of symmetry in the image

$$
S_{k}(\mathrm{X})=\left(\frac{1}{m \times n}\right) \frac{\sum(f(x, y)-M)^{3}}{S D^{3}}
$$

\subsubsection{Classification}

This is the crucial step in the whole process where all the data gathered is now put into various ML algorithms and final results are obtained stating the location and classification of the tumours. Some of the widely used algorithms are

\subsection{Multi Layer Perceptron}

This is a feed forward network where each node is considered as a neuron which has a non-linear activation function. This usually is made of three layers: input, hidden and output. A vanilla neural network is a MLP with only one hidden layer. Backpropagation is used for training the network,

The output of this algorithm only depends on the current input. When a certain piece of data is processed, then the connection weights are changed. The rate of error after each step is calculated and the machine tries to minimize this rate of error by trying to find the optimum weights associated with each. edge.

\section{Literature Survey}

We discuss a few strategies below:

\subsection{Median Filter}

This filter comes under the category of non-linear filters and is widely used typically in filtering "Gaussian", "salt and pepper" and "random" categories of noise, mainly because in some particular scenarios it helps keeps the edges of the image being processed intact.

The strategy of a median filter is to put the median of the $\mathrm{NxN}$ neighbouring values(called as window) in the place of the centre pixel. The window is the few preceding and following entries in a 1D model, whereas in a higher dimensional model, it corresponds to the radius of the region.

In practice, this means replacing the grey level of a particular pixel with the grey level median of the window of that particular pixel.

In order to calculate the median before replacing a particular pixel, the values in the window are first sorted, then then median is calculated.

$\mathrm{f}(\mathrm{x}, \mathrm{y})=\operatorname{median}\{\mathrm{g}(\mathrm{a}, \mathrm{b})\}$

where $(x, y)$ represent the pixel to be replaced and $(a, b)$ represent the window, centred around the pixel $(x, y)$.

\subsection{Gaussian High Pass filter}

Sharpening of the image can be achieved by using Gaussian high pass filters. This is done by passing the image through various high pass filters after the noise in the image has been eliminated[7]. The result of this would be an image with sharper boundaries than before. 
So basically, this is an operation to be done after the image is cleared of any noise, and not specifically to be used for clearing any "salt and pepper" noise in the image.

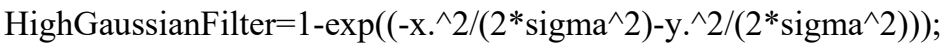

The processing speed to efficiency ratio is comparatively better than other methods of sharpening It might not preserve the brightness of the original image in some cases

\subsection{Morphology Dilation}

In a binary image, dilation refers to modify the foreground pixels by enlarging their boundaries, thus making the holes in them smaller.In a grayscale image[14], which is our use case, the images are functions which map an Euclidian grid into the set of Real numbers.

$$
(f \oplus \mathrm{b})(\mathrm{x})=\sup _{\mathrm{y} \in E}[\mathrm{f}(\mathrm{y})+\mathrm{b}(\mathrm{x}-\mathrm{y})]
$$

This also is an operation to be applied after eliminating the noise from an image, with the prime motivation to regularise the shape of the image and obtain principal connected components, which in our use case is the tumour region.

So the final image will enhance the smaller bright spots and eliminate the small dark spots.

When this is applied on a brain MRI, the end product will have the tumor will be visible in white color.

\subsection{Adaptive Histogram Equalization}

This is a strategy widely used to improve the local contrast in images while also enhancing the edges of the image[5].This method studies multiple histograms in which each belong to a unique section of the image, and used them to redistribute the lightness value within the image, by applying a transformation function (which is proportional to the $\mathrm{CDF}$ ) derived from a neighbourhood region onto each pixel[4].

The neighbourhood of pixels near the boundaries would not lie within the image, hence the image is to be extended by mirroring the pixel lines and columns and not simply copying them(which would cause a highly peaked neighbourhood histogram) maintaining consideration for the boundary of the image.

\subsection{Wiener Filter}

This is the optimal linear filter solving the problem of noise or blur in the image. The wiener filter can only supress noise but it can't expel it from the image[7]. As for the blur,

$G(u, v)=F(u, v) \cdot H(u, v)$

Where $\mathrm{H}$ and $\mathrm{F}$ are blurring function(sombrero function for focus blurring) and the Fourier transform of the perfect version of the image respectively. In practical experiments, it becomes hard to calculate the value of $\mathrm{H}$. These filters can't correct and image for which the value of $\mathrm{H}$ is 0 due to bandlimiting.

This technique is very efficient in terms of the mean square error.

$$
\mathrm{W}\left(f_{1}, f_{2}\right)=\frac{H^{*}\left(f_{1}, f_{2}\right) S_{x x}\left(f_{1}, f_{2}\right)}{\left|H\left(f_{1}, f_{2}\right)^{2}\right| S_{x x}\left(f_{1}, f_{2}\right)+S_{\eta \eta}\left(f_{1}, f_{2}\right)}
$$

where $S_{x x}\left(f_{1}, f_{2}\right), S_{\eta \eta}\left(f_{1}, f_{2}\right)$

are respectively power spectra of the original image and the additive noise.It uses both high pass and low pass filters for inverse filtering and compression operation respectively.

Various segmentation methods are discussed below:

\subsection{Watershed segmentation}

This strategy utilizes the concepts of image morphology. Pre-processed images are conditioned to edge detection techniques like canny edge detection. These detected edges are crucial for the segmentation[16].A seed point interior to the image is provided, which includes the background as a separate object.The image is treated as a topographic landscape which has multiple valleys and ridges which in the image are classified based on the gradient magnitude[16]. Each pixel is either marked part of a region or a watershed.

This is one of the most simple yet highly accurate segmentation method and is thus widely used mostly because of the low overhead it provides.

\subsection{Berkeley wavelet transform}

A threshold is selected and the image is converted into a binary form. Based on this threshold, pixels greater than that are mapped to white, and the pixels less in value are mapped to black[11]. Morphology erosion is then used to eliminate the white pixels and the black pixels are used for the MRI mask.

The BWT is a triadic wavelet transform. The mother wavelet transformation is a piecewise constant function[17]. The substitute wavelets from the mother wavelet are produced at various pixel positions in the two-dimensional plane through scaling and translation of the mother wavelet and it is shown in: 


$$
\beta_{\theta}^{\oplus}(\tau, s)=\frac{1}{s^{2}} \beta_{x}^{\oplus}\left(3^{s}(x-i), 3^{s}(y-j)\right)
$$

\subsection{K Means}

This is an unsupervised algorithm used on unlabeled data. This runs on the basic principle to reduce the sum of all the squared distances between the cluster center and all the points[3] that belong to that particular cluster.

$$
I=\sum_{j=1}^{k} \sum_{i=1}^{x}\left\|x_{i}^{(j)}-c_{j}\right\| \wedge 2
$$

The process involves selection of $\mathrm{N}$ number of centroids and then assigning all the points in the image to the nearest centroid. Then new centroids are calculated and the whole process is again cycled.The drawback of this method involves 'the random initialization' trap. This happens because of the random selection of centroids

\subsection{Neural Network backed segmentation}

There are many neural network based segmentation techniques like CNN, which involves mapping the whole image several pixels at a time until the moment it has mapped the whole image[13]. These pockets of pixels are called as filters. These apply several layers which are fully connected. An improvement of this is the FCN[20], which are much faster and whose output has a final layer with a large receptive field and number of channels which correspond to the number of classes.Ensemble learners have many weak-learners which take parts of the image and classify them[6], and then all these results are compiled and a. final output is given, thus achieving very high levels of accuracy and low error rate.

\subsection{Naïve Bayes algorithm}

This is a probabilistic classifier primarily based on the Bayes theorem. Supervised learning of the machine takes place. The Bayes theorem considers that the presence or absence of a particular object is unrelated to the presence or absence of another[8]. The same concept is used even by this algorithm[2]. Because this has a standard theorem in place, there is not much amount of learning involved in this and thus the time taken for the execution of this algorithm is blazing fast. The probability probability is calculated and used to estimate the posterior proababilty.

\subsection{Support Vector Machine}

This is a supervised learning technique which can scale form one-class to n-class classifications. The SVM kernel function basically divides a nonlinear dividing objective into a linear transformation.

Linear:-The simple form in which the training sets are linearly separated.Representation[16]:

$(k)=w^{\mathrm{p}} k+b$

Non Linear: This defines a hyperplane that is divided into two training classes as defined in:

$$
f(y)=z^{T} \emptyset(y)+b
$$

Where $\mathrm{Z}$ and $\mathrm{T}$ are hyperplane parameters. For a brain MRI which. Is our current use case, the kernel class separability and features selection maked this a perfect choice[10].But the downsides of SVM are that it's performance takes a hit when overlapping classes are thrown at it[19], and it takes a long time to train.

\section{Result Analysis [9]}

Table 1. Methods and evaluation percentages

\begin{tabular}{|c|c|c|c|}
\hline Study & Method & $\begin{array}{c}\text { Proposed Solution and } \\
\text { Preprocessing Approach }\end{array}$ & Evaluation \\
\hline Ali ARI\&Davut & CNN & ELM-LRF & Accuracy:97.18\% \\
\hline YannickSuteret al & $3 \mathrm{D}-\mathrm{CNN}$ & SVC & Accuracy:72.2\% \\
\hline YuexiangLi\&Linlin & CNN & MvNet and SPNet & Accuracy: $88.00 \%$ \\
\hline Dong Nie et al & $3 \mathrm{D}-\mathrm{CNN}$ & Multichannel CNN & Accuracy:90.66\% \\
\hline JaveriaAminrt et al & Random Forest Class & GWF,HOG,SFTA & Accuracy:90.00\% \\
\hline LinaChato\&Shahram & CNN Linear Desc & SVM,KNN,Tree & Accuracy:68.8\% \\
\hline Virupakshappa & Adaptive ANN & Modified Level set App & Accuracy:98.00\% \\
\hline
\end{tabular}




\section{Conclusion}

This Paper focuses on the various approaches that can be taken to detect and classify brain tumors using Machine Learning and all the various steps involved in it. Some algorithms like CNN and SVM standout with their capabilities and algorithms like Bayes standout with their amazing speeds. The author during this survey has observed that no single method during any step of the process is perfect, but instead using hybrid methods gives promising results.

Probability based methods and statistics backed methods, both seem to flourish under different conditions, but some techniques fail miserably when there is more noise in the images. With this ever changing and fastmoving domain of research it concludes that no one method can give perfect results but modification and matching multiple methods can surely help the process.

\section{References}

[1] Manasavi Sharma, Chetan Marwaha "Brain Tumor Detection using Image Segmentation Techniques on MRI Images", International Journal of Innovative Technology and Exploring Engineering, 2020.

[2] Sanjay Kumar C K, H. D. Phaneendra "Classification of Tumors in Brain MRI Images With Hybrid of Global and Local DWT Features using Decision Tree", International Journal of Innovative Technology and Exploring Engineering, 2019.

[3] Dr. Michael J. Garbade "Understanding K-means Clustering in Machine Learning”,2018.

[4] E.Philip Amburn John D,Austin "Adaptive histogram equalization and its variations" 2020.

[5] Jyothi Shetty and Pawan S Jogi "Study on different region- based object Detection models applied to Live Video Stream and Images Using Deep Learning", Proceedings of the International Conference on ISMAC in Computational Vision and BioEngineering 2018 (ISMAC-CVB).

[6] E.Philip Amburn John D, Austin "link.springer.com".

[7] Ranjeet Kaur, Amit Doegar "Localization and Classification of Brain Tumor using Machine Learning \& Deep Learning Techniques",International Journal of Innovative Technology and Exploring Engineering, 2019.

[8] Methodokogy Zhang, Yu-Dong \& Wu, Lenan \& Wang, Shuihua. (2011) "Magnetic Resonance Brain Image Classification by an Improved Artificial Bee Colony Algorithm. Progress In Electromagnetics Research”. 116. 65-79. 10.2528/PIER11031709.

[9] Muhammad Waqas Nadeem , Mohammed A. Al Ghamdi 3, Muzammil Hussain 2 ,Muhammad Adnan Khan 1, Khalid Masood Khan 1, Sultan H. Almotiri 3 and Suhail Ashfaq Butt 4, 2020 "Brain Tumor Analysis Empowered with Deep Learning: A Review, Taxonomy, and Future Challenges".

[10] Nick Efford "Morphological Image Processing www.cs.auckland.ac.nz > ImageProcessing-html “) topic4.

[11] Nilesh Bhaskarrao Bahadure, Arun Kumar Ray, Har Pal Thethi. "Image Analysis for MRI Based Brain Tumor Detection and Feature Extraction Using Biologically Inspired BWT and SVM", International Journal of Biomedical Imaging, 2017.

[12] Nilesh Bhaskarrao Bahadure, Arun Kumar Ray, Har Pal Thethi. "Comparative Approach of MRI-Based Brain Tumor Segmentation and Classification Using Genetic Algorithm",Journal of Digital Imaging, 2018.

[13] Springer Science and Business Media LLC "Proceedings of the International Conference on IOT, Social, Mobile, Analytics and Cloud in Computational Vision and Bio-Engineering 2018 (ISMAC-CVB)", Springer Science and Business Media LLC,2018.

[14] IOP Publishing IOP Conf. Series "First International Conference on Advanced Algorithms and Control Engineering IOP Publishing IOP Conf. Series: "Journal of Physics: Conf. Series 1087 (2018) 062032 doi :10.1088/1742-6596/1087/6/062032 .

[15] Springer Science "RITA 2018", Proceedings of the 6th International Conference on Robot Intelligence Technology and Applications, Springer Science and Business Media LLC, 2020.

[16] Bernhard Preim, Charl Botha, "Segmentation in Visual Computing for Medicine" (Second Edition), Elsevier, 2014.

[17] Sevasti-Zoki Karakozoglou, Nathalie Henrich Bernardoni, Christophe d'Alessandro, Yannis Stylianou "Automatic glottal segmentation using local-based active contours and application to glottovibrography" 2015.

[18] Sevasti-Zoi Karakozoglou, Nathalie Henrich,Christophe d Alessandro, Yannis Stylianou. "Automatic glottal segmentation using localbased active contours and application to glottovibrography", Speech Communication, 2012.

[19] Springer Science " Soft Computing for Sustainability Science", Springer Science and Business Media LLC, 2018.

[20] Di.Univr.It “ Region-based Segmentation - documenti > matdid > matdid125113. 